\title{
Parálisis facial en fase aguda secundaria al síndrome de Tolosa Hunt: reporte de un caso
}

\author{
Jenniffer Alfonso Cuellar ${ }^{1}$ \\ https://orcid.org/0000-0002-7402-318x \\ Daniela Lizcano Medina \\ https://orcid.org/0000-0002-5306-4556 \\ Universidad Manuela Beltrán, Bogotá, Colombia. \\ Fecha de recepción: 13 de diciembre de 2018 \\ Fecha de aprobación: 30 de mayo de 2019
}

\begin{abstract}
Resumen
El síndrome de Tolosa Hunt corresponde a una inflamación granulomatosa idiopática que genera una parálisis facial y oculomotora ipsilateral, trastornos pupilares e hipoestesias y/o hiperalgesia en la zona inervada por la rama oftálmica del nervio trigémin. La literatura es escasa frente al manejo fisioterapetico en estos pacientes, por tal razón es necesario generar una propuesta basada en las estrategias convencionales y actuales para el manejo de pacientes con parálisis facial secundaria a este síndrome. Caso Clínico: mujer de 20 años que presenta parálisis facial secundaria al síndrome de Tolosa Hunt en quien se evidencia asimetría facial, compromiso en los movimientos del ojo, debilidad de la musculatura facial, escala House Brackmann reporta parálisis total, se encuentran puntos gatillos activos en hemicara comprometida y región cervical. Se observa una postura antálgica y compromiso de pares craneales III, IV, V, VI y VII. Intervención: Se realizan 12 sesiones de fisioterapia con el objetivo de disminuir dolor mejorando la movilidad, recuperar la función motora de la musculatura facial, mejorar la vascularización y la conducción nerviosa. Resultados: Posterior a la intervención fisioterapéutica se evidencian óptimos resultados frente a la movilidad, la fuerza, la simetría facial, la sensibilidad y la postura. Discusión: La literatura actual expone técnicas para el manejo de la parálisis facial que generan en el paciente efectos significativos frente a su recuperación motora principalmente. Es importante incluir dentro del manejo fisioterapéutico la aplicación de electroterapia que ha mostrado resultados en el manejo de las parálisis faciales acelerando la recuperación respecto al manejo convencional.
\end{abstract}

Palabras Clave: Parálisis facial; fisioterapia; parálisis.

${ }^{1}$ Correo de contacto: jalfonso0219@gmail.com

DOI: 10.30788/RevColReh.v18.n2.2019.371

Esta obra se encuentra bajo licencia internacional CC BY 4.0 Institución editora: Escuela Colombiana de Rehabilitación 


\title{
Facial paralysis in secondary acute phase to Hunt Tolosa syndrome: report of a case.
}

\begin{abstract}
The Tolosa Hunt syndrome corresponds to an idiopathic granulomatous inflammation that generates ipsilateral facial and oculomotor paralysis, pupillary disorders and hypoesthesia and / or hyperalgesia in the area innervated by the ophthalmic branch of the trigeminal nerve. Literature is scarce compared to physiotherapeutic management in these patients, for this reason it is necessary to generate a proposal based on conventional and current strategies for the management of patients with facial paralysis secondary to this syndrome. Clinical case: 20-year-old woman with facial paralysis secondary to Tolosa Hunt syndrome in whom facial asymmetry, compromised eye movement, facial muscle weakness, House Brackmann scale reported total paralysis, trigger points active compromised hemicara and cervical region. An antalgic posture and commitment of cranial nerves III, IV, V, VI and VII is observed. Intervention: 12 sessions of physiotherapy are carried out with the aim of reducing pain by improving mobility, recovering the motor function of the facial muscles, improving vascularization and nerve conduction. Results: After the physiotherapeutic intervention, optimal results are shown in terms of mobility, strength, facial symmetry, sensitivity and posture. Discussion: The current literature exposes techniques for the management of facial paralysis that generate significant effects in the patient against motor recovery mainly. It is important to include within the physiotherapeutic management the application of electrotherapy that has shown results in the management of facial paralysis, accelerating recovery from conventional management.
\end{abstract}

Keywords: Facial paralysis; physical therapy especialty; paralysis.

\section{Introducción}

El síndrome de Tolosa-Hunt (STH) se define como una inflamación granulomatosa idiopática del seno cavernoso o de la fisura orbitaria superior (Aguirre, Zúñiga \& Barrera 2014). Este síndrome fue descrito por primera vez en 1954 por Eduardo Tolosa, neurocirujano español, quien reportó el caso de un hombre de 47 años con dolor retro orbitario unilateral asociado a disfunción ipsilateral de los nervios oculomotores y de la rama oftálmica del trigémino. La anatomía patológica demostró una reacción inflamatoria granulomatosa que infiltró la porción intra cavernosa de la carótida interna y de los nervios craneales adyacentes (Tolosa, 1954). Posteriormente en 1961, William Edgar Hunt, neurólogo norteamericano, presenta 6 casos compatibles con la descripción previa de Tolosa, y su relación con los primeros criterios diagnósticos, adicionalmente señala la buena respuesta de los pacientes al tratamiento esteroidal. (Hunt, 1961). Cinco años después se identificó la entidad nosológica como el síndrome de Tolosa -Hunt (Smith 1966) y en 1988 es aceptado como entidad nosológica por la sociedad internacional de Clasificación de Cefaleas (Díaz \& González, 2009). Actualmente la literatura nacional e internacional presenta mínimos reportes de casos que den cuenta del manejo fisioterapéutico en este tipo de pacientes, por lo que cobra relevancia este artículo, ya que con la presentación de este caso exitoso, se muestra un avance importante frente a la rehabilitación de pacientes que cursan con este síndrome, aportando en su manejo interdisciplinario, previniendo la aparición de secuelas que comprometen el movimiento principalmente de la musculatura facial e impactando en los resultados a corto plazo, a demás de proporner un manejo oportuno desde el objeto de estudio de la profesión con estrategias convencionales e innovadoras, basado en la interpretación de la patología, su origen y semiología. 
Como objetivo del presente estudio se plantea presentar los resultados obtenidos frente a la propuesta de intervención fisioterapéutica en un paciente con parálisis facial en fase aguda secundaria al Síndrome de Tolosa Hunt, aplicada en 12 sesiones de intervención.

\section{Descripción del síndrome de Tolosa Hunt.}

El síndrome de Tolosa-Hunt es una inflamación granulomatosa idiopática del seno cavernoso que afecta predominantemente a los pares craneales tercero y quinto en su recorrido por el interior de este, manifestándose con frecuencia en forma de oftalmoplejía dolorosa (Serralta, Torrecillas, Soler, Ibáñez \& Gómez, 2013). La International Headache Society determinó en el 2004 los criterios diagnósticos de este síndrome.

Tabla 1.

Criterios diagnóstico del síndrome de Tolosa-Hunt ICHD-II.

\begin{tabular}{cl}
\hline Criterio & \multicolumn{1}{c}{ Descripción } \\
\hline A & Uno o más episodios de dolor unilateral persistente si no recibe tratamiento \\
B & $\begin{array}{l}\text { Parálisis de uno o más de los siguientes: III par, IV par y/o VI par y/o demostración de } \\
\text { granuloma por IRM o biopsia. }\end{array}$ \\
C & $\begin{array}{l}\text { Parálisis oculomotora que coincide con la etapa aguda del dolor o dentro de las } 2 \text { semanas } \\
\text { de aparición del mismo }\end{array}$ \\
D & $\begin{array}{l}\text { Dolor y paresias oculomotoras que resuelven después de } 72 \text { horas de tratamiento adecuado } \\
\text { con corticoides } \\
\text { Otras causas fueron excluidas (tumorales, vasculitis, meningitis basal, sarcoidosis, diabetes }\end{array}$ \\
E & y migraña oftalmopléjica).
\end{tabular}

Fuente: D Aguirre, G Zúñiga \& L Barrera. (2014). Tolosa-Hunt Syndrome: Case Report And Literature Review. Acta Neurológica Colombiana, 30(4), p. 348.

Los pares craneales oculomotores pueden afectarse en cualquier combinación posible, siendo el III par craneal el más frecuentemente afectado, las inervaciones simpáticas y parasimpáticas pueden verse afectadas siendo evidente por las alteraciones pupilares presentes en el examen de estos pacientes (Martínez, Casasco, Pendre, De Bonis \& Berner, 2010). El III par o motor ocular común, corresponde a un complejo nuclear mesencefálico de localización medial que yace ventral al acueducto de Silvio. El nervio óptico puede estar comprometido, lo cual indica que el proceso inflamatorio involucra el ápex orbitario. Otros pares craneales, que no cruzan esta región afectada, pueden comprometerse como la rama maxilar superior e inferior del V par o el nervio facial (Martínez et al., 2010).

Este síndrome se acompaña de una parálisis facial periférica que según Giannaula (2010), “corresponde a la lesión del núcleo del facial y sus aferencias de lesión homolateral. Los hallazgos semiológicos corresponden a la ubicación topográfica de la lesión. La lesión a nivel del agujero estilomastoideo presenta compromiso motor, afectando los movimientos voluntarios y de la gesticulación. Por tanto, el paciente no puede fruncir el ceño, la hendidura palpebral es mayor en el lado afectado y no es posible ocluir el ojo homolateral. Al solicitarle que lo haga se observa la rotación del globo ocular hacia arriba (signo de Bell). Al solicitar al paciente que mire hacia arriba el globo ocular puede ascender más que el ojo sano. El parpadeo está abolido. Puede presentar lagrimeo 
(epifora) por imposibilidad de absorber las lágrimas debido a la separación del conducto lagrimal de la conjuntiva. El surco nasogeniano se encuentra borrado. La boca se presenta traccionada hacia el lado sano por acción del orbicular de los labios contralateral. La saliva puede derramarse por la comisura labial. El paciente no puede protruir los labios, lo cual le impide silbar, y al intentar soplar, la mejilla del lado afectado se hinchará más por la parálisis del buccinador. No podrá contraer el cutáneo del cuello.”(p.23).

Etimológicamente no presenta una causa clara, se atribuye su aparición a un agente patógeno externo que puede ser de tipo viral o bacteriano. Como se sabe, la presencia de este tipo de agentes genera una activación de la cascada inflamatoria, que en este caso se produce específicamente en el seno cavernoso y/o en la fisura orbitaria superior, pudiendo comprometer estructuras adyacentes como el origen de pares craneales, principalmente III, IV, V, VI y secundariamente el VII par.

Con el presente caso se da a conocer una propuesta de intervención terapéutica, en una paciente con parálisis facial en fase aguda, secundario al síndrome de Tolosa Hunt, con el fin de brindar información sobre las estrategias fisioterapéuticas que permiten una mejoría significativa en la evolución clínica de la paciente.

\section{Fisiopatología del síndrome de Tolosa Hunt.}

Con el fin de comprender los mecanismos fisiopatológicos que reporta la literatura y por los cuales se origina la patología provocando sus signos y síntomas, se realizará una breve explicación de cada uno de estos teniendo en cuenta cuál es su repercusión en el movimiento corporal humano.

En primera instancia, hay un cambio en la permeabilidad del tejido que produce cambios en el flujo vascular y en el calibre de los pequeños vasos como son vénulas y arteriolas, esto se da gracias al acúmulo de eritrocitos en la zona afectada producidos por el aumento del flujo sanguíneo secundario a la vasodilatación, en la que conjuntamente hay activación de los leucocitos, en especial los macrófagos, quienes son los encargados de iniciar el proceso de fagocitosis y de la activación de los mediadores inflamatorios como el ácido araquidónico, responsable de la reducción de pro- inflamatorios como las interleucinas 1 y 6 , y el aumento de anti-inflamatorios como el factor de necrosis tumoral-beta (Fonseca, 2013).

El síndrome de Tolosa Hunt se asocia a un componente de granulomatosis, el cual se genera por el fracaso de la fagocitosis del cuerpo extraño que activa los linfocitos $\mathrm{T}$ que produciendo un aumento de los macrófagos epitelioides, denominados así por su parecido aspecto a las células epiteliales debido a que su núcleo es más grande y alargado que el de los macrófagos normales, estos se rodean por un collar de leucocitos mononucleares y células plasmáticas formando un granuloma, indicador principal de inflamación crónica (Granados-Reyes, Soriano-Redondo \& Durán-Ferreras, 2012). Con base en lo anterior, esta inflamación compromete el origen de los pares craneales II, III, IV, V, VI y VII, disminuyendo la velocidad de conducción de las fibras nerviosas, dando origen a la alteración sensitiva maxilar y a la parálisis oftálmica y facial. 
La lesión que se genera en el nervio facial puede ser provocada por factores como traumas o tumores, edema secundario a inflamación. En la porción laberíntica del canal de Falopio, conducto por donde pasa el nervio, la arteria principal tiene menor diámetro que en las otras porciones y la ocupación espacial del nervio en dicho conducto es en promedio del $48 \%$ en el segmento laberíntico, $46 \%$ en el timpánico y $32 \%$ en el mastoideo, factores que podrían explicar el por qué en la parálisis facial periférica hay mayor compromiso isquémico e inflamatorio del nervio en la porción laberíntica (Rondón, 2009).

El nervio facial recorre un trayecto de $35 \mathrm{~mm}$ dentro del canal óseo, lo que lo hace más susceptible a la acción de procesos comprensivos e infecciosos de variada naturaleza, interrumpiendo su impulso nervioso conllevando al bloqueo total de sus funciones; posterior a una lesión, el nervio mantiene su excitabilidad por más de 96 horas, con los axones recibiendo energía de las células de Schwann, produciendo transformaciones histológicas como la fibrilación axonal con posterior desaparición de los axones y edematización de las células de Schwann destruyendo la mielina por fagocitosis, proceso llamado degeneración walleriana que ocurre entre los 15 a 20 días después de la lesión como cita Ferreira Bento, Vuono de Brito \& Menino Castillo (2004).

La degeneración walleriana consiste en la infiltración en el sitio de la lesión de células T, neutrófilos y macrófagos en los dos primeros días, donde las células de Schwann secretan citoquinas inflamatorias, factor de necrosis tumoral e interleucinas, estimulando la reabsorción y regeneración axonal. Los macrófagos penetran a los túbulos de las células de Schwann para degradar las vainas de mielina y fagocitar las dendritas, segregando lipoproteínas Y para compactar los lípidos del axón y los restos de mielina, lo cuales son entregados a las células de Schwann para su reutilización durante la regeneración (Gutiérrez-Mendoza, 2014).

Aproximadamente al tercer y cuarto día comienza un proceso de regeneración axonal de distal a proximal, algunas células de Schwann sobreregulan la codificación del RNAm segregando vainas de mielina, mientras que otras permanecen sin mielina, provocando así una cronicidad que puede conducir a la apoptosis, como cita Syroid et al. (1996).

Teniendo en cuenta lo anterior, según Arandicidia (2016) la agresión sufrida por el nervio desencadena los siguientes grados de lesión: a) neuropraxia: Existe apenas un bloqueo fisiológico capaz de causar parálisis, no llevando a una degeneración walleriana. Terminado el bloqueo se observa una regeneración completa de los axones sin secuelas; b) axonotmesis: La continuidad del nervio persiste, pero ha ocurrido degeneración walleriana; y c) neurotmesis: es la sección completa del nervio, no existiendo posibilidad de recuperación espontánea.

La lesión a nivel de los pares II, III, IV y VI se comportan fisiopatológicamente con los mismos mecanismos mencionados anteriormente, en el trayecto del origen de su localización en el foco inflamatorio del síndrome. Por otro lado el compromiso de V par craneal, se debe a una neuralgia, aunque los mecanismos fisiopatológicos siguen siendo controversiales, en este caso es válido inferir que la irritación crónica o el estiramiento de las fibras aferentes resulta de una disminución de la inhibición a nivel de los núcleos trigeminales, con pérdida de neuronas inhibitorias y disminución de la actividad de las fibras aferentes primarias gruesas, con la generación de potenciales ectópicos 
y repetitivos. Estos potenciales se transmiten por contacto de tipo efáptico (eléctrico) a las fibras amielínicas nociceptivas. La falta de modulación de estas fibras por la disminución de la inhibición segmentaria determina la descarga paroxística de las neuronas trigémino - talámicas de amplio rango, lo que finalmente resulta en percepción dolorosa. (Albert Díaz, Ruiz Ruiz, Linares Hernández \& Ruiz Ruiz, 1998).

Teniendo en cuenta todos los mecanismos fisiopatológicos anteriormente mencionados, desde el punto de vista fisioterapéutico basado en los dominios y categorías según la guía APTA en 2016 se verían comprometidos principalmente: Dominio neuromuscular $->$ Categoría integridad de pares craneales, categoría dolor y el dominio musculoesquelético -> Categoría desempeño muscular.

\section{Implicaciones Biomecánicas:}

Como se sabe, los nervios periféricos tienen propiedades mecánicas de elongación, tracción y elasticidad, en el momento de sufrir la lesión inflamatoria ya descrita dichas propiedades se ven alteradas debido al fenómeno de compresión que causa sobre la estructura, en este caso lenta y progresiva, impidiendo que el nervio se elongue llevándolo a un acortamiento y aumentando su sensibilidad a la tracción provocando secundariamente una ruptura (Miralles,1998); por consiguiente, la lesión mecánica es directamente proporcional a la lesión vascular con isquemia, ya que éstas modificaciones mecánicas a la tracción provocan fibrosis epineural, aumentando la resistencia y disminuyendo la elasticidad nerviosa (Miralles, 1998). Esto se verá reflejado en la disminución o impedimento de la recepción del impulso a la placa motora y la aferencia sensorial, provocando a nivel motor la pérdida del tono muscular y la imposibilidad de contracción en la hemicara afectada y a nivel sensitivo hiper, hipo o disestesia según sea el caso.

Debido a la pérdida de contracción, la hemicara sana sufrirá un aumento del tono por hiperactividad gamma como ventaja mecánica, provocando puntos gatillo que contribuyen a la asimetría facial ya establecida. Dependiendo del caso particular se encontrará afección en músculos específicos, generalmente se ven comprometidos los músculos frontal, superciliar, orbicular de los ojos, piramidal de la nariz, caninos, ala de la nariz, cigomático mayor y menor, risorio, buccinador, orbicular de los labios, triangular de los labios y cuadrado de la barba, provocando imposibilidad de realizar la gesticulación facial de forma adecuada, dificultad para el proceso de alimentación e imposibilidad para pronunciar las palabras correctamente comprometiendo la función motriz facial de los usuarios afectados.

\section{Presentación del Caso}

Paciente género femenino de 20 años, quien acude al servicio de urgencias por presentar exacerbación de cefalea tensional de predominio izquierdo de un mes de duración, reporta que en las últimas horas presenta dolor en hemicara izquierda, pérdida de la fuerza muscular y asimetría facial, refiere dolor periorbicular y maxilar, en el momento tratada con esteroides. Es remitida al servicio de fisioterapia por presentar parálisis facial izquierda, pupila hiperreactiva midriática izquierda, ptosis palpebral, desviación de la comisura labial, historia clínica reporta que en las últimas horas se diagnosticó posible compromiso del seno cavernoso izquierdo con un diagnóstico diferencial de 
lesión granulomatosa del seno cavernoso, se encuentra reporte de TAC que arroja como diagnóstico síndrome de Tolosa Hunt sin descartar trombosis del seno cavernoso izquierdo y de angiotomografía que confirma compromiso granulomatosos del seno cavernoso con posible trombosis, además reporta compromiso de los pares III, IV, V, VI y VII .

\section{Valoración fisioterapéutica}

Paciente en quien en la valoración fisioterapéutica se observa con rubor y aumento de la temperatura en hemicara izquierda, las líneas bipupilar, ótica y oclusiva no se encuentran paralelas lo que confirma asimetría facial, en cuanto a la movilidad articular se evaluaron movimientos fisiológicos tanto activos como pasivo en donde no se evidencia oclusión ocular del párpado superior izquierdo. Llama la atención el nulo movimiento ocular en ojo izquierdo, hay desviación marcada de la comisura labial izquierda, la amplitud del movimiento presenta hipomovilidad en la hemicara izquierda, la paciente no ejecuta movimientos del globo ocular izquierdo, ni tampoco rota dentro de la cavidad orbitaria en torno a los tres ejes principales de movimiento, en evaluación de la movilidad articular de la columna cervical la paciente completa rangos de movimiento con presencia de dolor 10/10 de acuerdo a la EVA. Frente a la exploración muscular se encuentra con calificación de 0: ausencia de la funcionalidad en escala de Daniels para los músculos recto superior, recto inferior, recto medial, recto lateral, oblicuo mayor, y oblicuo motor; respecto a la actividad facial en hemicara izquierda, se encontró para el elevador del párapado superior y orbicular de los párpados calificación NF: es incapaz de abrir el ojo y el iris está casi completamente cubierto por el párapado, para el orbicular de los ojos, es incapaz de cerrar el ojo y el iris no esta completamente cubierto por el párpado y para el superciliar la calificación es 0 ya que no hay movimiento visible al fruncir el ceño, esta misma puntuación se mantiene para el músculo elevador de las cejas, el músculo piramidal y miltiforme, orbicular de los labios, bucinador, canino, risorio, cigomáticos, masetero, temporal, pterigoideo, borla de barba y triangular de los labios.

La paciente no presenta sincinesias, valoración por escala House Brackmann reporta grado VI parálisis total, pérdida del tono y ausencia de contracción. Se realiza palpación en músculos faciales derechos encontrando en el frontal, masetero, pterigoideo puntos gatillos activos, además se encuentran puntos álgicos en trapecios fibras superiores, esplenios de la cabeza, escalenos y esternocleidomastoideo bilateral. Presenta dolor calificado $8 / 10$ en escala análoga visual, especialmente en la palpación en musculatura facial y de 10/10 en región cervical. Se observa una postura antálgica, hiperlordosis cervical, leve inclinación hacia la derecha, hombros descendidos, protruidos, flancos disminuidos, codos semiflexionados, alineación glútea, rodillas semiflexionadas, base de sustentación amplia, presenta compromiso de pares craneales III, IV, V, VI y VII, evidenciando ptosis palpebral izquierda, desviación del globo ocular sin ningún movimiento del globo ocular, discoria y anisocoria, reflejo consensual positivo, parálisis del músculo masetero, mandíbula desviada hacia la izquierda, se observa desviación de la comisura labial, paciente no es capaz de fruncir el ceño, existe asimetría labial, no es capaz de contener el aire en la mejilla izquierda paciente presenta alteraciones en la percepción del gusto además refiere hiperalgesia con sensación de quemazón en rama maxilar que varía con la estimulación sensorial.

\section{Intervención fisioterapéutica}


Se realizaron 12 intervenciones, 3 semanales, fundamentadas en la propuesta que se presenta en la tabla 2. Cada intervención corresponde a una hora de duración. Para el manejo de esta paciente, teniendo en cuenta la fase en la que se encuentra de acuerdo al cuadro clínico descrito previamente, se plantean como objetivos terapéuticos principales el control de dolor e inflamación, la facilitación de conducción de nervios periféricos y promoción de la reeducación motriz, con esto pretendía atender la sintomatología propia de un proceso inflamatorio agudo, ,lograr simetría facial, recuperar la fuerza y funcionalidad en la musculatura facial, a demás prevenir la instauración de alteraciones posturales.

Tabla 2.

Propuesta de intervención fisioterapéutica de acuerdo con la evidencia científica.

\begin{tabular}{|c|c|c|c|}
\hline $\begin{array}{c}\text { Objetivo } \\
\text { terapéutico }\end{array}$ & $\begin{array}{c}\text { Medidas } \\
\text { terapeuticas }\end{array}$ & Prescripción & Evidencia \\
\hline \multirow[t]{2}{*}{$\begin{array}{l}\text { Disminuir dolor e } \\
\text { inflamación } \\
\text { en la zona cervical } \\
\text { permitiendo } \\
\text { mayor calidad } \\
\text { en la ejecución de } \\
\text { movimientos }\end{array}$} & $\begin{array}{l}\text { Masaje } \\
\text { terapéutico }\end{array}$ & $\begin{array}{l}\text { Conducta: técnicas de } \\
\text { Terapia Manual } \\
\text { Tipo: masaje de tejido } \\
\text { conectivo terapéutico y } \\
\text { movilización y } \\
\text { manipulación de tejidos } \\
\text { blando } \\
\text { Intensidad: Suave } \\
\text { Región : Trapecios } \\
\text { Velocidad: Lenta } \\
\text { Dirección: de origen a } \\
\text { inserción } \\
\text { Medio: aceite mineral } \\
\text { Duración: } 10 \text { minutos } \\
\text { Frecuencia: Al iniciar cada } \\
\text { sesión de Ft. }\end{array}$ & $\begin{array}{l}\text { Fernández de las Peñas \& Fernández Carnero } \\
\text { (2004) realizan un estudio en donde proponen la } \\
\text { realización del masaje transverso profundo en la } \\
\text { zona de los trapecios buscando disminuir el dolor } \\
\text { miofascial. Encontraron en } 10 \text { sujetos estudiados una } \\
\text { mejoría significativa frente al umbral del dolor. } \\
\text { La terapia manual incluye según Gross y cols como } \\
\text { lo cita Capó-Juan,(2015) cinco categorías: } \\
\text { manipulaciones, movilizaciones pasivas, masaje, } \\
\text { técnicas de tejidos blandos y terapias de } \\
\text { movilización neuromuscular. Según muestra una } \\
\text { revisión reciente la terapia manual se ha considerado } \\
\text { como el conjunto de técnicas más eficaces en la } \\
\text { inactivación de Puntos gatillo. }\end{array}$ \\
\hline & $\begin{array}{l}\text { Vendaje } \\
\text { neuromuscular } \\
\text { en trapecio } \\
\text { fibras. }\end{array}$ & $\begin{array}{l}\text { Conducta: Prescripción y } \\
\text { adaptación de dispositivos } \\
\text { de asistencia y equipos de } \\
\text { protección y soporte. } \\
\text { Modalidad: Protección } \\
\text { Tipo: vendaje } \\
\text { neuromuscular } \\
\text { Técnica: de inserción a } \\
\text { origen muscular } \\
\text { Tensión: } 10 \% \text { - } 25 \% \\
\text { Frecuencia: realizar cambio } \\
\text { de vendaje entre } 3 \text { y } 5 \text { días } \\
\text { Duración tratamiento: } 12 \\
\text { sesiones } \\
\text { Localización: Trapecio } \\
\text { fibras superiores. }\end{array}$ & $\begin{array}{l}\text { Se propone la utilización de kinesiotaping como una } \\
\text { estrategia terapéutica alterna que permita reducir el } \\
\text { dolor, aumentar los rangos de movimiento y el } \\
\text { fortalecimiento de la musculatura cervical, } \\
\text { especialmente en trapecios fibras superiores, de } \\
\text { acuerdo a Villota \& Fernández (2018) en su estudio } \\
\text { proponen la colocación del kinesiotaping en la } \\
\text { región cervical con el fin de lograr los objetivos } \\
\text { anteriormente mencionados, por su parte en una } \\
\text { revisión sistemática realizada por Carrasco-Mendoz } \\
\text { (2014) quien busca examinar en la evidencia } \\
\text { científica sobre efectos analgésicos para el manejo } \\
\text { del dolor musculoesquelético, concluyendo que el } \\
\text { vendaje neuromuscular es una técnica eficaz para } \\
\text { disminuir el dolor. }\end{array}$ \\
\hline
\end{tabular}




\begin{tabular}{ll}
\hline $\begin{array}{l}\text { Provocar } \\
\text { mayor }\end{array}$ & $\begin{array}{l}\text { Aplicación de } \\
\text { crioterapia }\end{array}$ \\
vascularización de & sobre la \\
la zona favoreciendo & hemicara \\
conducción nerviosa & izquierda
\end{tabular}

Aplicación de Termoterapia sobre la hemicara izquierda
Conducta: Agentes físicos
Modalidad: Crioterapia
Tipo: Masaje con hielo
Técnica: toques rápidos en
dirección de fibras
musculares
Duración: 7 a 10 minutos en
cada sesión
Frecuencia: Todos los días x

5 días

Localización: musculatura facial

Conducta: Agentes físicos

Modalidad: Termoterapia

Tipo: Paquete caliente

Duración: 10 minutos en

cada sesión

Frecuencia: Todos los días a

partir del 6 día.

Localización: musculatura facial
El efecto de la crioterapia se fundamenta en los cambios que se demuestran a nivel de la electrofisiología neuromuscular; ya que se ve asociado a la disminución local de la temperatura cutánea que produce un retraso, tanto en la apertura como en el cierre de los canales de sodio, produciendo un enlentecimiento de las corrientes de sodio responsables de la despolarización de las fibras nerviosas, lo que se traduce finalmente en una reducción de la de conducción nerviosa del axón, el hecho de tener un periodo refractario más largo, ocasionando una disminución del potencial de acción, que sumado a la disminución de la frecuencia de descarga de los nociceptores, explicarían el aumento del umbral del dolor y el potencial efecto hipoalgésico atribuido a la crioterapia (Capó-Juan, 2015).

Se propone el uso de compresas húmedas durante 10 minutos con el objetivo de mantener la vascularización y los cambios móviles a la espera del influjo nervioso promoviendo la relajación muscular y la preparación para la realización de estiramientos musculares, ya que la sensibilidad del territorio del trigémino no presente ninguna alteración. (Matos, 2011).

\begin{tabular}{l}
\hline Promover una \\
reeducación \\
motriz que le \\
permitan \\
recuperar la \\
función normal de \\
la musculatura, \\
proporcionando \\
mayor control y \\
calidad de \\
movimiento.
\end{tabular}

Movilizaciones

Conducta: Ejercicio

terapéutico

Modalidades:

Entrenamiento de la fuerza, resistencia para músculos faciales.

Tipo: pasivos, activos asistidos, activos, activos resistidos progresivamente. Programa estandarizados de ejercicios relacionados con la mímica facial.

Técnica: Resistidos (para la realización de activos resistidos)

Medio: resistencia manual Frecuencia: 5 veces $\mathrm{x}$ semana

Duración: 20 minutos diarios- 12 sesiones Localización: músculos faciales

Conducta: Ejercicio terapéutico Modalidad: Ejercicios de

Programa de flexibilidad La Touche, Escalante, Linares, \& Mesa (2008) refieren que la prescripción de este tipo de actividades se utiliza con el objetivo de promover la simetría facial, mejorar la expresión facial, mejorar la alimentación y la comunicación, además de prevenir o evitar la aparición de sincinesias cuando se está ejecutando algún ejercicio. Algunas de las estrategias que plantea son: aplicar resistencia sólo en movimientos aislados realizar ejercicios de estiramientos del lado afectado en la musculatura de la mímica que pueda estar implicada en las sincinesias, enseñar al paciente a percibir la tensión y la relajación muscular, y enseñarle métodos para reducir la tensión cuando esta aparezca. Los ejercicios planteados están directamente relacionados con movimientos de expresiones emocionales faciales se recomienda prescribir no más de 10 repeticiones por ejercicio.

Uno de los principios de la facilitación Neuromuscular (FNM) es el reflejo del estiramiento $\mathrm{y}$ de la resistencia para promover la actividad y aumentar la fuerza muscular de los músculos de la cara. La presión y resistencia adecuadas tienen en cuenta facilitar el movimiento. Algunos de los principios generales tratamiento de la cara son los movimientos faciales son ejercitados con tareas funcionales. 


\begin{tabular}{|c|c|c|}
\hline $\begin{array}{l}\text { fino y específico } \\
\text { de ejercicios } \\
\text { faciales para } \\
\text { rehabilitar la } \\
\text { sincinesia. }\end{array}$ & $\begin{array}{l}\text { Conducta: Prescripción y } \\
\text { adaptación de dispositivos } \\
\text { de asistencia y equipos de } \\
\text { protección y soporte. } \\
\text { Modalidad: Protección } \\
\text { Tipo: vendaje } \\
\text { neuromuscular } \\
\text { Técnica: de origen a } \\
\text { inserción muscular } \\
\text { Tensión: } 15 \% \text { - } 35 \% \text { de } \\
\text { tensión } \\
\text { Frecuencia: realizar cambio } \\
\text { de vendaje entre } 3 \text { y } 5 \text { días } \\
\text { Duración tratamiento: } 12 \\
\text { sesiones } \\
\text { Localización: musculatura } \\
\text { facial. }\end{array}$ & $\begin{array}{l}\text { Alptekin (2017) propone como manejo terapéutico } \\
\text { en una paciente con diagnóstico de parálisis facial } \\
\text { periférica House-Brackmann grado III la aplicación } \\
\text { del Kinesiotaping en el área nerviosa y muscular } \\
\text { afectada con el objetivo de principalmente la } \\
\text { neurofacilitación y el alivio del dolor edema, } \\
\text { logrando un efecto analgésico en el cuadro clínico de } \\
\text { la paciente. } \\
\text { Avendaño-Sosa, Sánchez-Ramón,Valier-Izquierdo, } \\
\text { Chapital-Solís, \& Arcos González (2015), } \\
\text { fundamentaron en su estudio la utilización del } \\
\text { vendaje neuromuscular desde el concepto de } \\
\text { estimulación tegumentaria, éste aplicado en parálisis } \\
\text { facial genera estímulo cutáneo proporcionando } \\
\text { aferencias constantes y duraderas hacia la corteza } \\
\text { sensorial primaria, lo que permite una mejor } \\
\text { integración del sistema somatosensorial, realizaron } \\
\text { un estudio descriptivo, longitudinal en un periodo de } \\
4 \text { meses en } 2 \text { pacientes con diagnóstico de Parálisis } \\
\text { Facial Periférica y encontraron que en ambos casos } \\
\text { la aplicación del Vendaje Neuromuscular condujo a } \\
\text { notables mejorías desde la primera semana de } \\
\text { aplicación, siendo un tipo de terapia que actúa las } 24 \\
\text { horas del día, permitiendo una constante } \\
\text { estimulación, misma que acorta el tiempo de } \\
\text { recuperación del paciente con Parálisis Facial. }\end{array}$ \\
\hline
\end{tabular}

\section{Resultados finales:}

Al realizar 12 intervenciones de fisioterapia, se evidencia en la paciente mejoría frente a la disminución de dolor e inflamación en la zona cervical y hemicara izquierda, hubo una notable recuperación en la actividad nerviosa de los pares craneales III, IV, V, VI Y VII, así como en la movilidad, la reeducación motriz y función motora de la musculatura ocular y facial, se logro simetría facial y se reeeducó la postura en la paciente. Se disminuye puntuación en escala de House Brackmann pasando de una parálisis total a diferencia obvia pero no desfigurante entre ambos lados de la cara. Estos resultados se describen con mayor detalle en la Tabla 3. 
Tabla 3.

Tabla comparativa de hallazgos sesión 1 y sesión 12.

\begin{tabular}{|c|c|}
\hline Valoración & Resultados sesión 1 \\
\hline & $\begin{array}{l}\text { Presenta dolor calificado } 8 / 10 \text { en escala } \\
\text { análoga visual, especialmente en la } \\
\text { palpación en musculatura facial y de } 10 / 10 \\
\text { en región cervical. }\end{array}$ \\
\hline Dolor & $\begin{array}{l}\text { Presencia de puntos gatillo en músculos } \\
\text { faciales derechos en el frontal, masetero, } \\
\text { pterigoideo, además se encuentran puntos } \\
\text { álgicos en trapecios fibras superiores, } \\
\text { esplenios de la cabeza, escalenos y } \\
\text { esternocleidomastoideo bilateral. }\end{array}$ \\
\hline Simetría facial & $\begin{array}{l}\text { Las líneas bipupilar, ótica y oclusiva no se } \\
\text { encuentran paralelas, hay desviación } \\
\text { marcada de la comisura labial izquierda. }\end{array}$ \\
\hline \multirow[t]{2}{*}{ Movilidad } & $\begin{array}{l}\text { No se evidencia oclusión ocular del párpado } \\
\text { superior izquierdo. nulo movimiento } \\
\text { ocular en ojo izquierdo No ejecuta } \\
\text { movimientos del globo ocular izquierdo, ni } \\
\text { tampoco rota dentro de la cavidad orbitaria } \\
\text { en torno a los tres ejes principales de } \\
\text { movimiento }\end{array}$ \\
\hline & $\begin{array}{l}\text { Movilidad articular de la columna } \\
\text { cervical la paciente completa rangos de } \\
\text { movimiento con presencia de dolor. }\end{array}$ \\
\hline
\end{tabular}

\section{Resultados sesión 12}

Se disminuyó el cuadro doloroso en la musculatura facial y cervical a 2/10 de acuerdo a la escala análoga visual.

No se encontraron puntos gatillo ni zonas de irritabilidad o inflamación facial y cervical.
Las líneas bipupilar, ótica y oclusiva se encuentran paralelas, sin desviación de la comisura labial izquierda.

Paciente realiza oclusión ocular del párapado superior izquierdo, realiza movimientos del globo ocular izquierdo y rota dentro de la cavidad orbitaria en los 3 ejes de movimiento.

Se conservan rangos de movimiento en columna cervical y se logran movimiento en todos los ángulos a nivel ocular, sin presencia de dolor.

En la exploración final se asigna clasificación en escala Daniels "F" para los músculos recto superior, rector inferior, recto medial, recto lateral, oblicuo mayor y menor.

Se generó aumento de acuerdo a la escala de Daniéls en los músculos

Elevador parapado superior, orbicular de los parpados, superciliar, el elevador de las cejas con una calificación de "NF", Músculo piramidal y multiforme, orbicular de los labios, buccinador, canino, risorio, cigomáticos, masetero frontal, orbicular de los labios, piramidal de la nariz, orbicular de los ojos, cigomático menor y mayor, buccinador, pterigoideo, risorio y masetero, pteriogoideo, borla de barba y triangular de labios con calificación 
de "FD" en escala Daniels. Se logra simetría facial, especialmente en la línea ótica y la línea bucal.

\begin{tabular}{|c|c|}
\hline Postura & $\begin{array}{l}\text { Se describen hallazgos encontrados en } \\
\text { plano anterior, posterior y lateral, leve } \\
\text { inclinación hacia la derecha, hombros } \\
\text { descendidos, protruidos, asimetría de masa } \\
\text { muscular de trapecios , flancos } \\
\text { disminuidos, codos semiflexionados, } \\
\text { hipercifosis, alineación espinas iliacas, } \\
\text { alineación glútea, rodillas semiflexionadas, } \\
\text { base de sustentación amplia. }\end{array}$ \\
\hline $\begin{array}{l}\text { Escala House } \\
\text { Brackmann }\end{array}$ & $\begin{array}{l}\text { Grado VI parálisis total, pérdida del } \\
\text { tono y ausencia de contracción. }\end{array}$ \\
\hline Pares craneales & $\begin{array}{l}\text { Compromiso de pares craneales III, IV, } \\
\text { V, VI y VII, evidenciando ptosis palpebral } \\
\text { izquierda, desviación del globo ocular sin } \\
\text { ningún movimiento del globo ocular, } \\
\text { discoria y anisocoria, reflejo consensual } \\
\text { positivo, reflejo fotomotor negativo, } \\
\text { parálisis del músculo masetero, mandíbula } \\
\text { desviada hacia la izquierda, se observa } \\
\text { desviación de la comisura labial, paciente } \\
\text { no es capaz de fruncir el ceño, existe } \\
\text { asimetría labial, no es capaz de contener el } \\
\text { aire en la mejilla izquierda paciente } \\
\text { presenta alteraciones en la percepción del } \\
\text { gusto además refiere hiperalgesia con } \\
\text { sensación de quemazón en rama maxilar } \\
\text { que varía con la estimulación sensorial. }\end{array}$ \\
\hline
\end{tabular}

Alineación de la cabeza respecto al tronco, Hombros nivelados y simetría de trapecios, flancos disminuidos, codos en extensión, columna alineada, alineación de espianas iliacas, línea glútea, rodillas en semiflexión, base de sustentación amplia.

Grado III / VI disfunción moderada, diferencia obvia pero no desfigurante entre ambos lados de la cara.

Se observa que la paciente eleva el parpado superior, contrae la pupila, gira el globo ocular hacia arriba, abajo, adentro, afuera , contrae la pupila, acomoda el ojo, rota el globo ocular, se observa simetria facial, levanta sutilmente la ceja izquierda, frunce el ceño, muestra los dientes, cierra y apreta ojos, percibe diferentes sabores. 

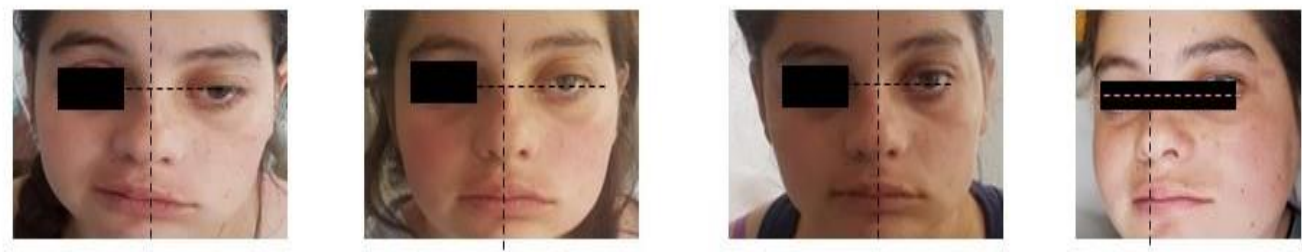

Sesión 1

Sesión 4
Sesión 7

Figura 1. Registro Fotográfico de los resultados del manejo de la parálisis facial secundario al Síndrome de Tolosa Hunt en 12 sesiones de fisioterapia.

\section{Discusión}

El síndrome de Tolosa Hunt es de etiología desconocida, representa aproximadamente el 9\% de los casos de inflamación orbitaria idiopática. Como sintomatología principal se destaca dolor intenso en región periorbitaria y retroocular, en algunas ocasiones como en este caso se acompaña de del compromiso de pares craneales, la literatura no reporta datos que permitan una asociación en esta sintomatología, sumado a esto la literatura nacional e internacional presenta mínimos reportes del manejo fisioterapéutico en este tipo de casos, razón por la que este estudio es de gran relevancia a nivel profesional y científico Zimmermann-Paiz, (2008).

En la actualidad los medios diagnósticos de mayor relevancia para este síndrome lo constituyen la resonancia magnética, la tomografía axial computarizada que permite detectar alteraciones vasculares y el compromiso del seno cavernoso, pruebas diagnósticas realizadas en la paciente que permitieron esclarecer el diagnóstico y confirmar las causas de la sintomatología, de esta manera se logró dar un adecuado manejo que permitió la evolución clínica y satisfactoria en la paciente.

La literatura actual expone técnicas convencionales y novedosas para el manejo de la parálisis facial, como la aplicación de medios físicos, la facilitación neuromuscular, la aplicación del kinesiotaping, la realización de técnicas manuales, que fueron aplicadas en la paciente logrando efectos significativos frente a su recuperación motora. Es importante incluir dentro del manejo fisioterapéutico la aplicación de electroterapia como la magnetoterapia, el láser y la terapia combinada, los cuales han mostrado resultados importantes en el manejo de las parálisis faciales acelerando la recuperación respecto al manejo convencional. Castillo, del Rio, García, Winter, \& Aguilera (2012). 
El uso del vendaje neuromuscular pacientes con parálisis facial favorece la modulación del tono de la musculatura facial y resulta útil, en casos en donde es necesario activar la musculatura periorbicular, peribucal y la función deglutoria (Avendaño-Sosa et al., 2015).

Se recomienda tomar este estudio como referente para el manejo fisioterapéutico en el manejo de pacientes que cursan con parálisis facial en fase aguda, secundario a diagnóstico clínico de síndrome de Tolosa Hunt, además se sugiere como referente en próximos estudios de investigación que fortalezcan científicamente el abordaje, manejo oportuno y apropiado de estos pacientes.

En conclusión, teniendo en cuenta que el síndrome de Tolosa-Hunt es una patología poco frecuente, se mostró la importancia de la intervención temprana desde terapia física en estos pacientes, lo cual da gran relevancia a nivel profesional y científico a este estudio. Se mostraron los resultados obtenidos en un corto plazo y en una fase aguda, de acuerdo al proceso de rehabilitación planteado desde el análisis del origen de la parálisis, brindando herramientas necesarias para demostrar el avance y mejoría en el estudio de caso.

Se logró evidenciar que la aplicación de técnicas como los medios físicos, la facilitación neuromuscular, el vendaje neuromuscular y técnicas manuales mostraron efectos significativos en cuanto a la realización de los diferentes acciones motoras de la musculatura facial, la asimetría facial. A su vez se realiza un aporte en cuanto a otras alternativas terapéuticas que sirven como apoyo en el momento de realizar este tipo de intervenciones, dejando así una experiencia aplicable al campo de la medicina de rehabilitación con grandes aportes basados en la evidencia.

\section{Agradecimientos.}

Las autoras manifiestan su agradecimiento a la paciente por aceptar la participación en este estudio de caso, a la Universidad Manuela Beltrán y a la Sub red Centro Oriente y en especial al Hospital de la Victoria por contribuir en la formación de fisioterapeutas y permitir el desarrollo científico de la profesión.

\section{Conflicto de Intereses}

Las autoras declaran no presentar ninguna relación de interés comercial o personal dentro del marco de la investigación que condujo a la producción del manuscrito.

\section{Colaboraciones}

Todos los autores han contribuido intelectualmente en la elaboración del documento.

\section{Referencias.}

Aguirre, D., Zúñiga, G. \& Barrera, L. (2014). Síndrome de Tolosa-hunt: Reporte de caso y revisión de la literatura. Acta Neurológica Colombiana, 30(4), 346-352. Doi: 10.22379/24224022155

Albert Díaz, J., Ruiz Ruiz, M., Linares Hernández, L., \& Ruiz Ruiz, C. (1998). Neuralgia del trigémino: fisiopatología, diagnóstico y tratamiento. Revista de Ciencias Médicas de Pinar del Río, 2(1), 2-14. Doi: 10.6018/194611 
Alptekin, D. Ö. (2017). Acupuncture and Kinesio Taping for the acute management of Bell's palsy: A case report. Complementary Therapies in Medicine, 35, 1-5. Doi: 10.1016/j.ctim.2017.08.013

Avendaño-Sosa, G. M., Sánchez-Ramón, J., Valier-Izquierdo, C. Y., Chapital-Solís, B. R., \& Arcos González, I. R (2015). Experiencia en el manejo de parálisis facial periférica con vendaje neuromuscular en el Centro de Rehabilitación y Educación Especial de Tabasco. Salud en Tabasco, 21(2-3). Doi: 10.1016/s0048-7120(06)74848-4

Capó-Juan, M. A. (2015). Síndrome de dolor miofascial cervical: revisión narrativa del tratamiento fisioterápico. Anales del Sistema Sanitario de Navarra, 38(1), 105-115. Doi: 10.4321/s113766272015000100011

Carrasco-Mendoz, A. (2014). Revisión sistemática sobre la efectividad de la aplicación del Kinesio Tape en el dolor musculoesquelético (Tesis de Grado). Universidad de Jaén. Ciencias de la Salud. Doi: 10.20986/resed.2016.3477/2016

Castillo, M. D., del Rio, M. S., García, A. D., Winter, G. P., \& Aguilera, I. G, (2012). Tratamiento combinado de campo magnético, láser, masaje y ejercicio en la parálisis facial periférica idiopática. Fisioterapia, 34(3): 99-104. Doi: 10.1016/j.ft.2011.11.002

Fernández de las Peñas, C., \& Fernández Carnero, J. (2004). Masaje de fricción transversal. Una alternativa terapéutica para el tratamiento del síndrome de dolor miofascial. Fisioterapia, 26(3), 126-133. Doi: 10.1016/s0211-5638(04)73094-1

Ferreira Bento R., Vuono de Brito R., Menino Castillo A.. (2004). Parálisis Facial Periférica. En Manual de Urgencias en Otorrinolaringología. 115-135.

Fonseca G. (2013). Fisiopatología de la inflamación. Recuperado de: academia.edu/16307039/Fisiopatologia_de_la_inflamaci\%C3\%B3n

Granados-Reyes, G. M., Soriano-Redondo, E., \& Durán-Ferreras, E. (2012). Síndrome de TolosaHunt tras un traumatismo ocular. Revista de Neurología, 54(12), 729-733. Doi: $10.33588 / \mathrm{rn} .5412 .2012050$

Gutiérrez-Mendoza I. (2014). Generalidades y fisiopatología de la compresión nerviosa. Orthotips,10(1); 9-14. Recuperado de https://www.medigraphic.com/cgibin/new/resumen.cgi?IDARTICULO=48933

La Touche, R., Escalante, A., Linares, M., \& Mesa, J. (2008). Efectividad del tratamiento de fisioterapia en la parálisis facial periférica. Revisión sistemática. Revista de Neurología, 46(12), 714-718. Doi:10.33588/rn.4612.2008066

Lietz-Kijak, D., Kopacz, Ł., Ardan, R., Grzegocka, M., \& Kijak, E. (2018). Assessment of the shortterm effectiveness of kinesiotaping and trigger points release used in functional disorders of the masticatory muscles. Pain Research and Management, Article ID 5464985, 7 pages. Doi:10.1155/2018/5464985

Martínez, D. F., Casasco, J. P., Pendre, N., De Bonis, C., \& Berner, S. I. (2010). Síndrome de TolosaHunt. Revista Argentina de Neurocirugía, 24(3): 111-115. Recuperdo de http://www.scielo.org.ar/pdf/ranc/v24n3/v24n3a06.pdf.

Matos, C. (2011). Paralisis facial periférica: O papel da medicina física e de reabilitação. Acta Médica Portuguesa, 24(4), 907-914. Recuperado de http://repositorio.chlc.minsaude.pt/handle/10400.17/920

Miralles, R. (1998). Biomecánica clínica del aparato locomotor. Barcelona, España: Masson S.A. 
Rondón, C. (2009). Parálisis facial periférica. Revista Médica Clínica Las Condes, 20(4), 528-535. Recuperado de http://www.clinicalascondes.com/areaacademica/pdf/MED_20_4/528_PARALISIS_FACIAL.pdf

Serralta San Martín, G., Torrecillas Narváez, D., Soler Rangel, L., Ibáñez Sanz, L., \& Gómez Cerezo, J. (2013). Sindrome de Tolosa-Hunt. Revista Española. 213(2), e11-e12. Doi: 10.1016/j.rce.2012.10.005

Syroid, D. E., Maycox, P. R., Burrola, P. G., Liu, N., Wen, D., Lee, K. F., Lemke, G., \& Kilpatrick T. J. (1996). Cell death in the Schwann cell IIneage and its regulation by neuroregulin. Proceedings of the National Academy of Sciences,93 (17)9229-9234. Doi: 10.1073/pnas.93.17.9229

Valdovinos, B. N., Morales, M. M., Boroevic, I. G., González, G. C., Cepeda, E. E. \& Dettoni, H. G. (2009). Síndrome de tolosa Hunt: Revisión a partir de un caso clínico. Revista memoriza.com, 5, 1-7. Doi: 10.1016/j.labcli.2008.12.001

Villota-Chicaíza, X. M. \& Fernández-Niño, J. A. (2018). Effects of neuromuscular taping as an independent or complementary method to physiotherapeutic treatment in the management of cervical pain. Revista de la Universidad Industrial de Santander Salud, 50(3), 195-204. Doi: 10.18273/revsal.v50n3-2018001

Zimmermann-Paiz, M. A. (2008). Síndrome de Tolosa-Hunt precedid de parálisis facial. Reporte de un caso. Revista Mexicana de Oftalmología, 82(1), 42-45. Recuperado de https://www.medigraphic.com/cgi-bin/new/resumenI.cgi?IDARTICULO=17489

\section{Notas:}

El presente artículo es el resultado del manejo fisioterapéutico que realizó una estudiante del programa de fisioterapia de la Universidad Manuela Beltrán, en su rotación en el Hospital de la Victoria bajo la orientación de la docente a cargo, a una paciente femenina de 20 años quien presentó cuadro clínico de parálisis facial originada por el síndrome de Tolosa Hunt. Este trabajo cumplió los requerimientos desde el punto de vista ético y por tanto el comité de ética en investigación de la Sub red Centro Oriente, Unidad de Servicios de Salud Santa Clara dan aprobación para su publicación.

\section{Información de autores:}

\section{Jenniffer Alfonso Cuellar}

Fisioterapeuta, MSc. en tecnologías digitales aplicadas en la educación.

Universidad Manuela Beltrán.

jalfonso0219@gmail.com

https://orcid.org/0000-0002-7402-318x

\section{Daniela Lizcano Medina}

Estudiante de Fisioterapia

Universidad Manuela Beltrán.

dlizcanomedina@gmail.com

https://orcid.org/0000-0002-5306-4556 